Revue d'histoire de l'enfance « irrégulière »

Le Temps de l'histoire

1 | 1998

La protection de l'enfance : regards

\title{
La convention collective de 1966 : chronique d'un rendez-vous impossible entre le privé et le public
}

\section{(2) OpenEdition \\ Journals}

Édition électronique

URL : http://journals.openedition.org/rhei/17

DOI : 10.4000/rhei. 17

ISBN : 978-2-7535-1638-0

ISSN : 1777-540X

Éditeur

Presses universitaires de Rennes

\section{Édition imprimée}

Date de publication : 15 novembre 1998

Pagination : 91-98

ISSN : 1287-2431

\section{Référence électronique}

«La convention collective de 1966 : chronique d'un rendez-vous impossible entre le privé et le public », Revue d'histoire de l'enfance « irrégulière » [En ligne], 1 | 1998, mis en ligne le 20 juin 2007, consulté le 04 décembre 2020. URL : http://journals.openedition.org/rhei/17 ; DOI : https://doi.org/10.4000/rhei.17 


\section{La convention collective de 1966 : chronique d'un rendez-vous impossible entre le privé et le public}

En préparant cet exposé, je me suis vite aperçu qu'il demeure encore des friches à explorer, des événements à élucider, des coïncidences à analyser. Puisque, on l'aura compris, je vais m'attarder sur les liens entre le secteur public et le secteur privé, dans ce que l'on nommera de façon générique "l'éducation spécialisée", il me semble que l'on tend encore à naviguer entre approximations et "images d'Épinal", faute notamment de recherches documentées, mais aussi faute d'une problématisation dégagée des pesanteurs expérientielles, dégagée de la croyance que, parce qu'on est du terrain, on connaît. Prenez donc ce que je vais avancer comme des propositions provisoires d'explication plus que comme des affirmations tranchées.

De fait, je voudrais risquer avec vous une prise de contre-pied, d'une certaine manière, contre l'idée d'une proximité telle entre versant "privé-associatif" et ses éducateurs patentés et secteur "public-Éducation surveillée”, puis PJJ, que vraiment il n'y avait qu'à tendre la main pour se rencontrer, qu'il n'y avait besoin que d'un peu de bonne volonté réciproque pour, par exemple, voir se fondre en un seul corps constitué les éducateurs de tous bords; corps qui aurait pu alors être affirmé, offensif, légitime donc.

L'affaire est plus complexe que cela et les apparences peuvent être trompeuses.

Ainsi, par exemple, ce n'est pas parce que, sur ce site de Vaucresson, en février 1968, se réunissaient nombre de directeurs d'écoles d'éducateurs - on dira du "privé" - élaborant les modalités de sélection, que des ponts étaient jetés; peut-être était-ce même l'inverse.

\author{
Alain \\ Vilbrod $^{(2)}$ \\ (1) Ce texte est la \\ communication de l'au- \\ teur aux Journées \\ d'études L'Éducation sur- \\ veillée aurait eu 50 ans, \\ Vaucresson, les 9 et 10 \\ novembre 1995. \\ (2) Maître de confé- \\ rence, Université de \\ Bretagne occidentale, \\ Brest.
}


(3) Dominique

Turbelin, La Justice a-t-elle besoin d'éducateurs ?, Érès, Toulouse, 1995.

(4) Michel Tachon, Recherche sur les processus de professionnalisation: analyse dans le secteur de l'enfance inadaptée au cours de la période 1945 1980, MIRE-AREPS, 1988.
Ce que je vais donc tenter, c'est de signaler que l'on avait, que l'on a affaire à deux sphères, à deux logiques, je dirai pour ma part à deux champs distincts qui, bien sûr, entretiennent des liens, mais qui n'en demeurent pas moins enserrés, chacun dans sa dynamique propre.

J'évoquerai la convention collective de 1966 régissant une large part du secteur privé. Ce n'est en fait qu'un analyseur, et d'ailleurs je déborderai assez largement ce point nodal pour notamment relever les enjeux de la création du diplôme d'État de 1967, comme par hasard quelques mois après, alors que le début de la gestation en remonte à septembre 1949.

Toutefois, avant cela, je rappellerai les lignes de force d'un secteur associatif éminemment agité, éminemment conflictuel, comme tout champ, éminemment original aussi, du moins dans la forme prise par les luttes internes, notamment entre corps professionnels.

J'ai lu avec un vif intérêt, on s'en doute, le livre de Dominique Turbelin. (3) J'y ai appris beaucoup, mais j'ai été surpris aussi. Il se dégage en effet, sur le point qui me préoccupe ici aujourd'hui, une vision que je ne partage pas complètement : d'un côté il y aurait les éducateurs spécialisés ayant désormais pignon sur rue, professionnels avérés, reconnus pour leur savoir-faire, et de l'autre des gens de métier pas tout à fait éducateurs, pas dotés du diplôme d'État qui certifie la profession, prisonniers d'un entre-deux, enclavés par des magistrats n'ayant aucun intérêt à laisser d'autres se tailler un territoire à égalité dans leur domaine de prédilection.

Cela renvoie à une citation, à mon sens un peu hâtive, de Michel Tachon affirmant qu'en deux décennies «les éducateurs spécialisés passent d'un statut mineur, où ils sont les tâcherons des notables associatifs, à celui d'un groupe professionnel ayant une place reconnue par l'État et institués comme acteurs à part entière du champ rééducatif ». ${ }^{(4)}$

Aux uns donc les bénéfices d'un domaine de compétence dans lequel leur statut occupe aujourd'hui une position dominante, aux autres les affres de la voie de garage et des tribulations de la déqualification, et cela faute d'avoir négocié l'aiguillage, faute d'avoir été au rendez-vous d'un grand soir d'où seraient nées une profession unifiée, une force sociale propre à damer le pion aux "psys" de tout acabit et aux magistrats de toute puissance. 
Je ne vois pas tout à fait les choses comme cela.

Les éducateurs de la PJJ sont-ils phagocytés, dominés, demi-professionnels, demi-portions en somme? A dire vrai je ne connais pas trop bien leur monde. Je connais mieux celui des éducateurs du secteur associatif, et je ne suis pas certain qu'ils puissent se prévaloir d'une reconnaissance pleine et entière, d'une technicité reconnue, bref d'une légitimité avérée.

Faut-il rappeler que le diplôme d'État date de 1967 et qu'il ne faudra pas attendre plus de trois ans pour voir apparaître des moniteurs-éducateurs inaugurant une prolifération verticale de mauvaise augure?

Les éducateurs spécialisés ont un statut, l'affaire est entendue, mais quitte à enfoncer des portes ouvertes, je pense que relier cela à la légitimité suppose un grand écart.

Le statut est affaire de droit ou, plutôt, moyen de cohésion sociale. Il régit l'ensemble des relations égalitaires et hiérarchiques d'un individu avec les autres membres de son groupe. On peut parfaitement avoir un statut et être en même temps, voire en raison même de ce statut, en position reléguée, assignée, dominée. Les aides-soignantes ont un statut. Faut-il prolonger les travaux de Claudine Herzlich ou d'Eliot Friedson pour rappeler combien elles sont aux ordres et fort peu légitimées dans leur fonction de contact avec le désarroi et la souffrance?

La légitimité est bien plus affaire de rôle que de statut, bien plus affaire de luttes de territoire que de savoirs du geste et de technicité éprouvée.

La légitimité, c'est toujours une conquête face aux autres, face à ceux d'à côté, bref la légitimité est "relationnelle", puisque liée aux entreprises de stratégie pour imposer sa place, son discours, ses représentations.

J'ai écrit ailleurs combien la légitimité était liée à la capacité d'un corps de métier à se constituer en profession, et ce, à partir de trois principes fondateurs : une technicité formalisée, un savoir reconnu, une capacité d'auto-organisation éprouvée. ${ }^{(5)}$

Je le rappellerai simplement en quelques mots, en signalant d'abord et avant tout que la reconnaissance est le fruit de luttes pour baliser son territoire, cultiver sa différence, se réserver pour soi tout seul des pratiques, des actes, quitte à rejeter dans l'amateurisme et le bricolage les concurrents.
(5) Alain Vilbrod,

"Écoutant : une profession?", Le Groupe Familial, nº148, 1995, p.5-17. 
(6) Pierre Bourdieu, "La force du droit. Éléments pour une sociologie du champ juridique", Actes de la recherche en sciences sociales, $\mathrm{n}^{\circ} 64$, 1986, p. 3-19.

(7) Francine MuelDreyfus. Le Métier d'éducateur, Paris, Minuit, 1983.
Quittons donc une vision consensuelle, proprette, mais finalement un peu molle. Imposer, s'imposer, en imposer nécessite une lutte permanente pour faire passer pour évidence des monopoles toujours plus ou moins arbitraires. La légitimité revient au corps professionnel qui est parvenu, fusse provisoirement, à clôturer son pré-carré, à ordonnancer les pratiques à la mesure de son savoir, quitte à développer pour cela des représentations sur l'intérêt suprême de l'usager, la pureté de la science experte dont il se prévaut, les garanties éthiques qu'il apporte. On pourrait dire que le couronnement de la légitimité est de pouvoir octroyer aux autres corps un statut taillé à la mesure des fonctions d'intermédiaires dominés que l'on attend d'eux.

Cela doit bien vous rappeler quelque chose; et là j’irai volontiers dans le sens de Dominique Turbelin. Par contre, on l'aura compris, je ne me risquerai pas à évoquer la légitimité pleine et entière des éducateurs spécialisés du secteur associatif.

Parlons un peu des premiers, quitte à ce que nous en débattions puisque, je l'ai dit, je ne suis pas vraiment en terrain de grande connaissance.

Nous n'allons tout de même pas, entre nous, avaliser le postulat d'un champ juridique qui serait dans une telle espèce d'apesanteur sociale qu'il serait ainsi autonome et indépendant des pressions et des enjeux proprement sociaux. Cela relèverait bel et bien d'une sorte d'idéologie professionnelle - j'allais dire confessionnelle - mettant en avant une rhétorique de la neutralité qui a fait long feu. Là aussi, bien entendu, il y a des luttes de concurrence pour, selon la formule de Pierre Bourdieu, " avoir le droit de dire le droit ". ${ }^{(6)}$ Luttes, mais limites à la lutte aussi, que l'on touche dès lors que les corps en jeu risqueraient de déflorer le fait qu'ils ne sont que des interprètes autorisés, et donc qu'il pourrait y avoir place pour d'autres corps concurrents. Ainsi, dans le champ juridique, il y a aussi une certaine entente entre lutteurs. Éric Pierre et Françoise Tétard ont ouvert un domaine passionnant, les juges pour enfants. Je crois me souvenir d'une réflexion de Francine Muel-Dreyfus ${ }^{(7)}$ sur leur création opportune au moment où il y a surproduction d'étudiants en droit. Et assurément il est passionnant d'étudier comment ils ont fait leur place, en mettant en avant leur pragmatisme, par exemple. 
J'ai été aussi frappé par la manière subtile dont ils ont opéré dans cette division du travail de domination symbolique en rénovant le métier d'éducateur, peut-être même - mais d'autres l'ont dit et le diront bien mieux que moi - en inventant "l'éducateur justice" moderne. Cet éducateur qui, parce qu'il a intérêt lui aussi à croire en la justice, s'impose sans doute certaines limites à sa subversion, tant il est bel et bien complice dans la production et la vente de biens et services juridiques.

Je perçois bien, comme le signale Dominique Turbelin, que les éducateurs et les magistrats ne sont pas dans un rapport d'égalité. Il y a effectivement division du travail. Les premiers sont des sondeurs appelés à côtoyer l'impur, mais aussi à appliquer de facto - et donc à faire le droit - en accomplissant la norme, en procédant au repérage, à la catégorisation, au marquage. Les seconds se nourrissent d'une certaine façon des situations concrètes rapportées, remontées par les premiers. Ils les enregistrent, ils les assimilent avant de s'en faire les exégètes et restent ainsi connectés, par corps interposé, avec un terrain dont ils se doivent de rester en prise, sauf à risquer que soit démasquée la lisibilité trop flagrante de l'arbitraire du jugement.

Jacques Bourquin ${ }^{(8)}$ rappelle à l'envi que le statut précède ici la formation, peut-être aussi en quelque sorte le métier, puisque, en avril 1945, on ne fait nullement référence à la formation de l'éducateur. Oubli ou urgence de créer un métier opportun, la question demeure, mais, précise-t-il aussitôt, "oubli" réparé puisque, dès 1947, Henri Michard organise au Centre d'observation de Savigny une première session de formation. Reste à en faire l'examen des contenus, qui s'annonce prometteur. De quel droit parlait-on? J'ai coutume d'avancer, pour ce qui concerne les éducateurs spécialisés, qu’on ne leur apprend pas la psychologie, mais la croyance aux savoirs des psychologues, ce qui n'est pas tout à fait la même chose. Pour l'heure, de quel droit? Comment opérait-on pour leur extorquer subtilement une complicité en leur donnant la maitrise minimale des textes canoniques? Comment théatralisait-on la dualité, voire l'antagonisme d'avec le personnel pénitentiaire rappelant les gaffes? Quand Sinoir bataille avec Joubrel ${ }^{(9)}$ sur les critères de sélection pour entamer une formation, en regardant avec
(8) Jacques Bourquin, "L'éducateur de l'Éducation surveillée au travers de l'histoire de sa formation", Cabiers du CRIV, $\mathrm{n}^{\circ} 4$, 1988, p. 87-100.

(9) Revue Rééducation, $\mathrm{n}^{\circ} 16$, juillet 1949 . 
(10) Alain Rugo, Analyse du développement des relations professionnelles dans le champ sanitaire et social au cours de la période 1950-1983, MIREAREPS, 1988. intérêt du côté des diplômes requis pour les instituteurs, quels enjeux? L'examen des contenus et des modalités, surtout des modalités qui souvent en disent plus long que les contenus formels, est incontournable. Que l'on pense aux travaux de Gisèle Morand-Durocher sur les assistants de service social par exemple.

Regardons maintenant un peu du côté du champ de l'initiative privée. Comme tout champ, comme tout secteur, sa structure renseigne bel et bien sur les rapports entre les corps, entre les groupes d'agents qui y interagissent. Rapports de force, mais aussi accord entre protagonistes s'imposant tacitement certaines limites aux subversions.

Que découvre t-on?

Avant tout, la velléité de se construire une entité coûte que coûte en délimitant son pré-carré, en se défiant des risques de dilution. Pour la sphère psychomédicale, les ordonnances de 1945 sur la Sécurité sociale consacrent tout un travail réalisé en amont. Elles intègrent les institutions de l'éducation spécialisée dans le "risque maladie", et non tel ou tel handicap ou inadaptation, et participent ainsi, elles aussi, de ce formidable blanc-seing à plusieurs volets qui va consacrer ce que Michel Chauvière appelle "le partage de pouvoir » entre les magistrats et les médecins.

La ligne de démarcation est tracée, et ce ne sont pas les timides tentatives de certains pour rattacher l'Éducation surveillée au ministère de la Santé qui pèseront pour quoi que ce soit. En effet, très rapidement, les acteurs du secteur privé vont avoir une préoccupation prioritaire : construire et consolider ce terrain de l'éducation spécialisée, encore mal délimité, mais déjà convoité.

Actuellement, je me penche sur les accords de 1958 entre l'UNAR (Union nationale des associations régionales de sauvegarde de l'enfance et de l'adolescence) et l'ANEJI (Association nationale des éducateurs de jeunes inadaptés). Ces accords matriciels visent bel et bien à résister aux tentatives d'avalement de la FEHAP (Fédération des établissements hospitaliers d'assistance privée) et, comme l'a bien montré Alain Rugo, ${ }^{(10)}$ l'UNAR et l'ANEJI entretiennent, le moins que l'on puisse dire, des rapports de connivence et de complicité pour faire pression sur l'État, sur un État dont ils semblent bien connaître, les uns et les autres, les arcanes. 
Les différends affichés ne doivent tromper personne : l'essentiel est

(11) Andrée Guiot, Les Problématiques de la sélection des éducateurs spécialisés, thèse d'État, Dijon, 1984. maître ; la cause commune fait l'union sacrée et l'on a même de la peine
quelquefois à dire qui est à l'UNAR et qui est à l'ANEJI, tant est grande la mobilité des acteurs.

Dans un tel contexte, il est clair que ce qui prime, c'est la consécration juridique et administrative d'une entité, clair aussi que l'union avec des partenaires comme l'Éducation surveillée n'est pas vraiment pensée, puisque ces armistices provisoires que sont les accords collectifs sont là avant tout pour renforcer l'architecture et la spécificité d'un domaine afin, entre autre, de rendre justement difficile, voire impossible, la relation avec les domaines voisins.

Ce processus sera long et, comme l'a bien expliqué Andrée Guiot, ${ }^{(1)}$ la question de la sélection à l'entrée en école, bac ou pas bac, renvoie à la volonté de ne pas faire comme les autres, de se singulariser, de structurer un marché et des institutions, de se constituer en groupe de pression.

Le diplôme d'État d'éducateur spécialisé viendra tard, tant il faudra du temps pour organiser l'espace des positions et spécifier ses interventions. Les accords de 1966 représentent un point nodal que, curieusement d'ailleurs, ne verra pas passer une ANEJI qui n'a pas encore pourtant tout à fait fini de faire son temps.

Curieuse convention, renvoyant là encore à un travail de division interne, à mille lieux de préoccupations d'alliance contre culture. Il fallait une convention... va pour une convention!

Mais cette convention ne fait, d'une part, que paraphraser des textes minimalistes, comme quand on recopie des statuts-type pour fonder une association 1901, tout en prenant, d'autre part, des libertés avec le code du travail, sur l'amplitude des horaires, par exemple. A la première lecture, on peut la percevoir peu favorable aux salariés, mais elle porte en fait la trace des libertés qu'ils se sont octroyées, parce que cela les arrangeait.

Si le secteur "Justice" est sans doute on ne peut plus fidèle à la Loi, "la grande", de la convention collective de 1966 se dégage l'empire des 
petites lois, des lois à part, des écarts à la règle. Elle est très imprécise, d'où l'accumulation des avenants, et renvoie continuellement à des règlements intérieurs qui, quand ils ne se révèlent pas complètement désuets, peuvent déroger à la loi, par exemple en infantilisant les handicapés, en édictant des interdits éventuellement contraire à la législation (interdiction de la consommation d'alcool, des relations sexuelles, etc.) au mépris même quelquefois de libertés individuelles que les éducateurs spécialisés découvrent béats, quand un juge les admoneste.

Quelle filiation y a t-il entre la convention collective de 1966 et le diplôme d'État de 1967? J'avoue demeurer encore dans l'expectative. Michel Chauvière écrit, dans la préface du livre de Dominique Turbelin, que "l'éducateur justice" a raté son rendez-vous avec le DE de 1967. Je voudrais bien sûr en savoir plus, tant il me semble que, s'il existe entre les deux secteurs des interférences, ne serait-ce que par "enfant de justice" interposé, on a bien affaire à deux champs enserrés chacun dans sa propre logique, sa propre dynamique, ses propres particularismes.

Il y a assurément de l'universel dans chacun des champs. Ainsi, éducateurs spécialisés et éducateurs "Justice" sont tous deux de ces corps subordonnés à d'autres qui font autorité, et sont donc plus ou moins inféodés. Les uns et les autres sont pour ainsi dire les derniers maillons de la chaîne de légitimité de violence symbolique, sont dans un métier d'entre-deux. Sans doute même leurs itinéraires familiaux et leurs aspirations à devenir éducateurs sont-ils très proches, mais ils sont partie prenante et partie agissante de deux secteurs ayant, d'une certaine manière, chacun leur propre loi. 